\title{
Differentiation of fatty acid composition of butter adulterated with lard using gas chromatography mass spectrometry combined with principal componenet analysis
}

\begin{abstract}
Butter is high priced product; as a consequence, butter can be subjected for adulteration with low price components such as lard. The presence of lard in any products is not allowed for Muslim and Jewish, therefore, its presence must be identified. Gas chromatography-mass spectrometry was successfully used to detect and discriminate butter from adulterated with lard. Results were presented in the form of chromatogram. Principal component analysis (PCA) was used to interpret the data and provided a good grouping of samples with $55.8 \%$ of the variation accounted for by PC 1 and $21.5 \%$ were accounted for by PC 2 . All the lard containing samples formed a separate group from the samples that were free of lard. This method can be developed into a rapid method for detecting the presence of lard in food samples for Halal authentication.
\end{abstract}

Keyword: Adulteration; Gas chromatography- mass spectrometry; Halal; Lard; Principal component analysis (PCA) 